\title{
Noncollapsing in mean-convex mean curvature flow
}

\author{
BEN ANDREWS
}

\begin{abstract}
We provide a direct proof of a noncollapsing estimate for compact hypersurfaces with positive mean curvature moving under the mean curvature flow: Precisely, if every point on the initial hypersurface admits an interior sphere with radius inversely proportional to the mean curvature at that point, then this remains true for all positive times in the interval of existence.
\end{abstract}

$53 \mathrm{C} 44 ; 58 \mathrm{~J} 35,35 \mathrm{~K} 93$

We follow Sheng and Wang [4] in defining a notion of "noncollapsing" for embedded hypersurfaces as follows: Recall that a hypersurface $M$ is called mean-convex if the mean curvature $H$ of $M$ is positive everywhere.

Definition 1 A mean convex hypersurface $M$ bounding an open region $\Omega$ in $\mathbb{R}^{n+1}$ is $\delta$-noncollapsed (on the scale of the mean curvature) if for every $x \in M$ there is an open ball $B$ of radius $\delta / H(x)$ contained in $\Omega$ with $x \in \partial B$.

It was proved in [4] that any compact mean-convex solution of the mean curvature flow is $\delta$-noncollapsed for some $\delta>0$. Closely related statements are deduced by Brian White in [6]. In both of these works the result is derived only after a lengthy analysis of the properties of solutions of mean curvature flow. The purpose of this paper is to provide a self-contained proof of such a noncollapsing result using only the maximum principle.

It is first necessary to reformulate the noncollapsing condition to allow the application of the maximum principle. Given a hypersurface $M=X(\bar{M})$, define a function $Z$ on $\bar{M} \times \bar{M}$ by

$$
Z(x, y)=\frac{H(x)}{2}\|X(y)-X(x)\|^{2}+\delta\langle X(y)-X(x), v(x)\rangle .
$$

Then we have the following characterization:

Proposition $2 M$ is $\delta$-noncollapsed if and only if $Z(x, y) \geq 0$ for all $x$ and $y$ in $\bar{M}$. 
Proof By convention we choose the unit normal $v$ to be outward-pointing, so that a ball in $\Omega$ of radius $\delta / H(x)$ with $X(x)$ as a boundary point must have centre at the point $p(x)=X(x)-(\delta / H(x)) v(x)$. The statement that this ball is contained in $\Omega$ is equivalent to the statement that no points of $M$ are of distance less than $\delta / H(x)$ from $p$ :

$$
0 \leq\|X(y)-p(x)\|^{2}-\left(\frac{\delta}{H(x)}\right)^{2}=\frac{2 Z(x, y)}{H(x)}
$$

for all $x$ and $y$ in $\bar{M}$. Since $H>0$ this is equivalent to the statement that $Z$ is nonnegative everywhere. The converse is clear.

The main result of this paper is the following:

Theorem 3 Let $\bar{M}^{n}$ be a compact manifold, and $X: \bar{M}^{n} \times[0, T) \rightarrow \mathbb{R}^{n+1}$ a family of smooth embeddings evolving by mean curvature flow, with positive mean curvature. If $M_{0}=X(\bar{M}, 0)$ is $\delta$-noncollapsed for some $\delta>0$, then $M_{t}=X(\bar{M}, t)$ is $\delta$ noncollapsed for every $t \in[0, T)$.

Proof By the Proposition, the Theorem is equivalent to the statement that the function $Z: \bar{M} \times \bar{M} \times[0, T) \rightarrow \mathbb{R}$ defined by

$$
Z(x, y, t)=\frac{H(x, t)}{2}\|X(y, t)-X(x, t)\|^{2}+\delta\langle X(y, t)-X(x, t), v(x, t)\rangle
$$

is nonnegative everywhere provided that it is nonnegative on $\bar{M} \times \bar{M} \times\{0\}$. We prove this using the maximum principle. For convenience we denote by $H_{x}$ the mean curvature and $v_{x}$ the outward unit normal at $(x, t)$, and we write

$$
d=|X(y, t)-X(x, t)|, \quad w=\frac{X(y, t)-X(x, t)}{d} \quad \text { and } \quad \partial_{i}^{x}=\frac{\partial X}{\partial x^{i}} .
$$

We compute the first and second derivatives of $Z$, with respect to some choices of local normal coordinates $\left\{x^{i}\right\}$ near $x$ and $\left\{y^{i}\right\}$ near $y$.

$$
\frac{\partial Z}{\partial y^{i}}=d H_{x}\left\langle w, \partial_{i}^{y}\right\rangle+\delta\left\langle\partial_{i}^{y}, v_{x}\right\rangle .
$$

From this we have the following:

\section{Lemma 4}

$$
v_{x}+\frac{d H_{x}}{\delta} w-\frac{1}{\delta} \frac{\partial Z}{\partial y^{q}} g_{y}^{q p} \partial_{p}^{y}=v_{y} \sqrt{1+\frac{2 H_{x}}{\delta^{2}} Z-\frac{1}{\delta^{2}}\left|\nabla_{y} Z\right|^{2}}
$$


Proof Equation (1) gives for each $i$

$$
0=\left\langle\partial_{i}^{y}, v_{x}+\frac{d H_{x}}{\delta} w\right\rangle-\frac{1}{\delta} \frac{\partial Z}{\partial y^{i}}=\left\langle\partial_{i}^{y}, v_{x}+\frac{d H_{x}}{\delta} w-\frac{1}{\delta} \nabla_{y} Z\right\rangle
$$

where

$$
\nabla_{y} Z=\frac{\partial Z}{\partial y^{k}} g_{y}^{k l} \partial_{l}^{y}
$$

Thus the vector $v_{x}+\left(d H_{x} / \delta\right) w-(1 / \delta) \nabla_{y} Z$ is normal to the hypersurface at $y$, and is a multiple of $v_{y}$. To complete the Lemma we compute the length of this vector:

$$
\begin{aligned}
\| v_{x} & +\frac{d H_{x}}{\delta} w-\frac{1}{\delta} \nabla_{y} Z \|^{2} \\
& =1+\left(\frac{d H_{x}}{\delta}\right)^{2}+2 \frac{d H_{x}}{\delta}\left\langle v_{x}, w\right\rangle+\frac{1}{\delta^{2}}\left|\nabla_{y} Z\right|^{2}-\frac{2}{\delta}\left\langle\nabla_{y} Z, v_{x}+\frac{d H_{x}}{\delta} w\right\rangle \\
& =1+\left(\frac{d H_{x}}{\delta}\right)^{2}+2 \frac{H_{x}}{\delta^{2}}\left(Z-\frac{d^{2} H_{x}}{2}\right)+\frac{1}{\delta^{2}}\left|\nabla_{y} Z\right|^{2} \\
& =1+\frac{2 H_{x}}{\delta^{2}} Z-\frac{1}{\delta^{2}}\left|\nabla_{y} Z\right|^{2},
\end{aligned}
$$

where we used the fact that $\nabla_{y} Z$ is in the tangent space at $y$, hence orthogonal to

$$
v_{x}+\frac{d H_{x}}{\delta} w-\frac{1}{\delta} \nabla_{y} Z
$$

Similarly we have (writing $h^{x}$ for the second fundamental form at $(x, t)$ )

$$
\frac{\partial Z}{\partial x^{i}}=-d H_{x}\left\langle w, \partial_{i}^{x}\right\rangle+\frac{d^{2}}{2} \nabla_{i} H_{x}+\delta d h_{i q}^{x} g_{x}^{q p}\left\langle w, \partial_{p}^{x}\right\rangle .
$$

Now the second derivatives:

$$
\frac{\partial^{2} Z}{\partial y^{i} \partial y^{j}}=H_{x}\left\langle\partial_{i}^{y}, \partial_{j}^{y}\right\rangle-d H_{x} h_{i j}^{y}\left\langle w, v_{y}\right\rangle-\delta h_{i j}^{y}\left\langle v_{y}, v_{x}\right\rangle,
$$

(4) $\frac{\partial^{2} Z}{\partial y^{i} \partial x^{j}}=-H_{x}\left\langle\partial_{j}^{x}, \partial_{i}^{y}\right\rangle+d\left\langle w, \partial_{i}^{y}\right\rangle \nabla_{j} H_{x}+\delta h_{j q}^{x} g_{x}^{q p}\left\langle\partial_{i}^{y}, \partial_{p}^{x}\right\rangle$,

$$
\begin{aligned}
\frac{\partial^{2} Z}{\partial x^{i} \partial x^{j}}= & H_{x}\left\langle\partial_{j}^{x}, \partial_{i}^{x}\right\rangle-d\left\langle w, \partial_{i}^{x}\right\rangle \nabla_{j} H_{x}+d H_{x} h_{i j}^{x}\left\langle w, v_{x}\right\rangle-d\left\langle w, \partial_{j}^{x}\right\rangle \nabla_{i} H_{x} \\
& +\frac{d^{2}}{2} \nabla_{j} \nabla_{i} H_{x}+\delta d \nabla_{j} h_{i q}^{x} g_{x}^{q p}\left\langle w, \partial_{p}^{x}\right\rangle-\delta h_{i j}^{x}-\delta d h_{i q}^{x} g_{x}^{q p} h_{p j}^{x}\left\langle w, v_{x}\right\rangle .
\end{aligned}
$$


Finally we compute the time derivative:

$$
\begin{aligned}
\frac{\partial Z}{\partial t}=d H_{x}\left\langle w,-H_{y} v_{y}+H_{x} v_{x}\right\rangle+ & \frac{d^{2}}{2}\left(\Delta H_{x}+H_{x}\left|h^{x}\right|^{2}\right) \\
& +\delta\left\langle-H_{y} v_{y}+H_{x} v_{x}, v_{x}\right\rangle+\delta d\left\langle w, \nabla H_{x}\right\rangle .
\end{aligned}
$$

We compute at a point $(x, y)$ with $y \neq x$. Choose local coordinates so that $\left\{\partial_{i}^{x}\right\}$ are orthonormal, $\left\{\partial_{i}^{y}\right\}$ are orthonormal, and $\partial_{i}^{x}=\partial_{i}^{y}$ for $i=1, \ldots, n-1$. Thus $\partial_{n}^{x}$ and $\partial_{n}^{y}$ are coplanar with $v_{x}$ and $v_{y}$.

Now compute

$$
\begin{array}{r}
\frac{\partial Z}{\partial t}-\sum_{i, j=1}^{n}\left(g_{x}^{i j} \frac{\partial^{2} Z}{\partial x^{i} \partial x^{j}}+g_{y}^{i j} \frac{\partial^{2} Z}{\partial y^{i} \partial y^{j}}+2 g_{x}^{i k} g_{y}^{j l}\left\langle\partial_{k}^{x}, \partial_{l}^{y}\right\rangle \frac{\partial^{2} Z}{\partial x^{i} \partial y^{j}}\right) \\
=d H_{x}\left\langle w,-H_{y} v_{y}+H_{x} v_{x}\right\rangle+\frac{d^{2}}{2}\left(\Delta H_{x}+H_{x}\left|h^{x}\right|^{2}\right)+\delta\left\langle-H_{y} v_{y}+H_{x} v_{x}, v_{x}\right\rangle \\
+\delta d\left\langle w, \nabla H_{x}\right\rangle-n H_{x}+d H_{x} H_{y}\left\langle w, v_{y}\right\rangle+\delta H_{y}\left\langle v_{y}, v_{x}\right\rangle-n H_{x}-d H_{x}^{2}\left\langle w, v_{x}\right\rangle \\
+2 d\left\langle w, \nabla H_{x}\right\rangle-\frac{d^{2}}{2} \Delta H_{x}-\delta d\left\langle w, \nabla H_{x}\right\rangle+\delta H_{x}+\delta d\left\langle w, v_{x}\right\rangle\left|h^{x}\right|^{2} \\
+2(n-1) H_{x}+2\left\langle\partial_{n}^{x}, \partial_{n}^{y}\right\rangle^{2} H_{x}-2 d g_{x}^{i k} g_{y}^{j l}\left\langle\partial_{k}^{x}, \partial_{l}^{y}\right\rangle\left\langle w, \partial_{j}^{y}\right\rangle \nabla_{i} H_{x} \\
-2 \delta\left(H_{x}-h_{n n}^{x}+\left\langle\partial_{n}^{x}, \partial_{n}^{y}\right\rangle^{2} h_{n n}^{x}\right) \\
=Z\left|h^{x}\right|^{2}+2 d\left\langle w, \partial_{k}^{x}-\left\langle\partial_{k}^{x}, \partial_{l}^{y}\right\rangle g_{y}^{l j} \partial_{j}^{y}\right\rangle g_{x}^{k i} \nabla_{i} H_{x}-2\left(H_{x}-\delta h_{n n}^{x}\right)\left(1-\left\langle\partial_{n}^{x}, \partial_{n}^{y}\right\rangle^{2}\right) .
\end{array}
$$

The second term on the last line can be rewritten in terms of the first derivatives of $Z$ using Equation (2): This gives

$$
\nabla_{j} H_{x}=\frac{2}{d^{2}} \frac{\partial Z}{\partial x^{j}}+\frac{2}{d}\left\langle w, H_{x} \partial_{j}^{x}-\delta h_{j p}^{x} g_{x}^{p q} \partial_{q}^{x}\right\rangle .
$$

Also we observe that $\partial_{n}^{x}-\left\langle\partial_{n}^{x}, \partial_{n}^{y}\right\rangle \partial_{n}^{y}=\left\langle\partial_{n}^{x}, v_{y}\right\rangle v_{y}$. Therefore at any critical point of $Z$ we have

$$
\begin{aligned}
\frac{\partial Z}{\partial t}=\sum_{i, j=1}^{n}\left(g_{x}^{i j} \frac{\partial^{2} Z}{\partial x^{i} \partial x^{j}}+g_{y}^{i j} \frac{\partial^{2} Z}{\partial y^{i} \partial y^{j}}+2 g_{x}^{i k} g_{y}^{j l}\left\langle\partial_{k}^{x}, \partial_{l}^{y}\right\rangle \frac{\partial^{2} Z}{\partial x^{i} \partial y^{j}}\right) \\
+\left|h^{x}\right|^{2} Z+2\left(H_{x}-\delta h_{n n}^{x}\right) Q
\end{aligned}
$$

where $Q=2\left\langle w, v_{y}\right\rangle^{2}\left\langle\partial_{n}^{x}, v_{y}\right\rangle^{2}-\frac{2 \delta}{d H_{x}}\left\langle w, v_{y}\right\rangle\left\langle\partial_{n}^{x}, v_{y}\right\rangle\left\langle\partial_{n}^{x}, \partial_{n}^{y}\right\rangle\left\langle\partial_{n}^{y}, v_{x}\right\rangle-\left\langle\partial_{n}^{x}, v_{y}\right\rangle^{2}$.

To simplify this we use Equation (1) to write $\left\langle\partial_{n}^{y}, v_{x}\right\rangle=-\left(d H_{x} / \delta\right)\left\langle w, \partial_{n}^{y}\right\rangle$. The first two terms in $Q$ then become

$$
2\left\langle w, v_{y}\right\rangle\left\langle\partial_{n}^{x}, v_{y}\right\rangle\left(\left\langle w, v_{y}\right\rangle\left\langle\partial_{n}^{x}, v_{y}\right\rangle+\left\langle\partial_{n}^{x}, \partial_{n}^{y}\right\rangle\left\langle w, \partial_{n}^{y}\right\rangle\right)=2\left\langle w, v_{y}\right\rangle\left\langle\partial_{n}^{x}, v_{y}\right\rangle\left\langle w, \partial_{n}^{x}\right\rangle,
$$


so we have

$$
Q=\left\langle\partial_{n}^{x}, v_{y}\right\rangle\left(2\left\langle w, v_{y}\right\rangle\left\langle w, \partial_{n}^{x}\right\rangle-\left\langle\partial_{n}^{x}, v_{y}\right\rangle\right)=\left\langle\partial_{n}^{x}, v_{y}\right\rangle\left\langle v_{y}, 2\left\langle\partial_{n}^{x}, w\right\rangle w-\partial_{n}^{x}\right\rangle .
$$

For convenience we write $\rho=\sqrt{1+\left(2 H_{x} / \delta^{2}\right) Z}$. Substituting for $v_{y}$ in the second factor using Lemma 4 we find

$$
\begin{aligned}
Q & =\left\langle\partial_{n}^{x}, v_{y}\right\rangle\left\langle v_{y}, 2\left\langle\partial_{n}^{x}, w\right\rangle w-\partial_{n}^{x}\right\rangle \\
& =\frac{\left\langle\partial_{n}^{x}, v_{y}\right\rangle}{\rho}\left\langle v_{x}+\frac{d H_{x}}{\delta} w, 2\left\langle\partial_{n}^{x}, w\right\rangle w-\partial_{n}^{x}\right\rangle \\
& =\frac{2\left\langle\partial_{n}^{x}, v_{y}\right\rangle\left\langle\partial_{n}^{x}, w\right\rangle}{\rho d \delta}\left(\delta d\left\langle w, v_{x}\right\rangle+\frac{d^{2} H_{x}}{2}\right) \\
& =\frac{2\left\langle\partial_{n}^{x}, v_{y}\right\rangle\left\langle\partial_{n}^{x}, w\right\rangle}{\rho d \delta} Z \\
& =\frac{2 H_{x}\left\langle\partial_{n}^{x}, w\right\rangle^{2}}{\rho^{2} \delta^{2}} Z .
\end{aligned}
$$

Thus Equation (7) becomes

$$
\begin{aligned}
\frac{\partial Z}{\partial t}=\sum_{i, j=1}^{n}\left(g_{x}^{i j} \frac{\partial^{2} Z}{\partial x^{i} \partial x^{j}}+g_{y}^{i j} \frac{\partial^{2} Z}{\partial y^{i} \partial y^{j}}\right. & \left.+2 g_{x}^{i k} g_{y}^{j l}\left\langle\partial_{k}^{x}, \partial_{l}^{y}\right\rangle \frac{\partial^{2} Z}{\partial x^{i} \partial y^{j}}\right) \\
& +\left(\left|h^{x}\right|^{2}+\frac{4 H_{x}\left(H_{x}-\delta h_{n n}^{x}\right)}{\delta^{2}+2 H_{x} Z}\left\langle\partial_{n}^{x}, w\right\rangle^{2}\right) Z .
\end{aligned}
$$

Since the coefficient of $Z$ is a smooth function which is bounded on $(M \times M) \backslash\{x=y\}$, the maximum principle implies that $Z$ remains nonnegative if initially nonnegative ( $Z$ is zero on the diagonal $\{y=x\}$ ).

Remarks (1) The computation is valid for curve-shortening flow of a convex curve.

(2) The estimate implies curvature pinching, ie $H_{x} g-\delta h^{x} \geq 0$.

(3) We made no use of the sign assumption on $\delta$, so the result also holds for negative $\delta$. This proves "exterior noncollapsing", ie the hypersurface remains outside the ball of radius $|\delta| / H_{x}$ which touches the tangent plane at $x$ on the exterior.

(4) The latter implies lower curvature pinching, ie $H_{x} g+|\delta| h^{x} \geq 0$.

(5) The same computation shows that $Z$ remains nonpositive if initially nonpositive. This applies in the case where $M$ is convex, and proves that if $M$ is contained in the ball of radius $\delta / H_{x}$ which touches the tangent plane at $x$ for every $x$ at the initial time, then this remains true for positive times. In this situation this implies curvature pinching, ie $H_{x} g-\delta h^{x} \leq 0$. 
(6) In the latter case the conclusion is much stronger than pointwise curvature pinching: It shows that the inradius and circumradius are both comparable to the reciprocal of the mean curvature at every point, and consequently that the mean curvatures at different points are comparable. The curvature pinching then implies that principal curvatures at different points are also comparable. This allows a very simple proof of convergence of convex hypersurfaces to spheres under mean curvature flow, recovering both Huisken's theorem [3] for $n \geq 2$ and Gage and Hamilton's theorem [1;2] for $n=1$.

(7) If the assumption of positive mean curvature is dropped, the conclusion still holds if we replace the mean curvature $H$ by any positive solution $f$ of the equation $\partial f / \partial t=\Delta f+\|A\|^{2} f$. In particular, this applies to prove a noncollapsing result if the initial hypersurface is star-shaped (see [5]).

Acknowledgement This research was partly supported by Discovery Projects grants DP0985802 and DP120102462 of the Australian Research Council.

\section{References}

[1] ME Gage, Curve shortening makes convex curves circular, Invent. Math. 76 (1984) 357-364 MR742856

[2] M E Gage, R S Hamilton, The heat equation shrinking convex plane curves, J. Differential Geom. 23 (1986) 69-96 MR840401

[3] G Huisken, Flow by mean curvature of convex surfaces into spheres, J. Differential Geom. 20 (1984) 237-266 MR772132

[4] W Sheng, X-J Wang, Singularity profile in the mean curvature flow, Methods Appl. Anal. 16 (2009) 139-155 MR2563745

[5] K Smoczyk, Starshaped hypersurfaces and the mean curvature flow, Manuscripta Math. 95 (1998) 225-236 MR1603325

[6] B White, The size of the singular set in mean curvature flow of mean-convex sets, J. Amer. Math. Soc. 13 (2000) 665-695 MR1758759

Mathematical Sciences Institute, Australian National University ACT 0200, Australia

ben.andrews@anu.edu . au

http://www . maths.anu.edu.au/ andrews/

Proposed: Tobias H Colding

Received: 31 July 2011

Seconded: John Lott, Gang Tian

Revised: 30 January 2012 